\title{
BIOCHEMICAL ASPECTS OF CORONAVIRUS REPLICATION
}

Luis Enjuanes, Fernando Almazán, Isabel Sola, Sonia Zúñiga, Enrique Alvarez, Juan Reguera, and Carmen Capiscol*

\section{INTRODUCTION}

Extensive morphological and biochemical changes occur in coronavirus (CoV)infected cells. Nevertheless, there is a limited knowledge of the biochemical events occurring in host cells and in the biochemistry of the infection. Infections by CoVs cause alterations in host cells in transcription and translation patterns, in the cell cycle, in the cytoskeleton, and in apoptosis pathways. In addition, in the host, CoV infection may cause inflammation, alterations of the immune response, of cytokine and chemokine levels, of interferon (INF)-induced gene expression and of stress responses, and modification of coagulation pathways. This chapter will focus on selected biochemical aspects of $\mathrm{CoV}$ replication and transcription with special attention to the interaction between cell and viral factors.

\section{INFLUENCE OF VIRAL AND CELLULAR PROTEINS IN COV REPLICATION}

\subsection{Nuclear Localization of $\mathrm{CoV}$ Proteins}

There are at least three $\mathrm{CoV}$ proteins that have been localized within the nucleus of infected cells: nucleoprotein $(\mathrm{N}), 3 \mathrm{~b}$, and nsp1 (Table 1). The nucleolus has been implicated in many aspects of cell biology that include functions such as ribosomal rRNA synthesis and ribosome biogenesis, gene silencing, senescence, and cell cycle regulation..$^{1-5}$ The nucleolus contains different factors including nucleolin, fibrillarin, spectrin, B23, rRNA, and ribosomal proteins S5 and L9. Viruses interact with the nucleolus and its antigens; viral proteins co-localize with factors such as nucleolin, B23, and fibrillarin and cause their redistribution during infection. ${ }^{2} \mathrm{~N}$ proteins from $\mathrm{CoV}$ genus $\alpha$

\footnotetext{
*Centro Nacional de Biotecnología, CSIC, Darwin, 3, Cantoblanco, 28049 Madrid, Spain.
} 
Table 1. NiV proteins in the nucleus of infected cells.

\begin{tabular}{|c|c|c|}
\hline PROTEIN & VIRUS & REFERENCE \\
\hline \multirow[t]{6}{*}{$\mathrm{N}$} & IBV & Chen, et al., 2003 \\
\hline & MHV & Wurm, et al., 2001 \\
\hline & SARS-CoV & Timani, et al., 2004 \\
\hline & TGEV & Wurm, et al., 2001 \\
\hline & PRRSV & Rowland, et al.,1999 \\
\hline & EAV & Tijms, et al., 2002 \\
\hline $3 b$ & SARS-CoV & Yuan, et al., 2005 \\
\hline nsp1 & EAV & van de Meer, et al., 1999 \\
\hline
\end{tabular}

(transmissible gastroenteritis virus, TGEV), ${ }^{6} \beta$ (mouse hepatitis virus, MHV, and severe and acute respiratory syndrome coronavirus, SARS-CoV), ${ }^{6-8}$ and $\gamma$ (infectious bronchitis virus, IBV), ${ }^{9}$ and also from two arteriviruses [porcine respiratory and reproductive syndrome virus (PRRSV) and equine arteritis virus (EAV) ${ }^{10,11}$ localize in the nucleolus, and this may be a common feature among nidovirus $\mathrm{N}$ proteins that influences host cell proliferation. ${ }^{6}$ The association of $\mathrm{N}$ protein with the nucleus may be cell dependent. In fact, until now, $\mathrm{N}$ protein has been located in the nucleus of LLC-PK1 and Vero cells transfected with plasmids expressing $\mathrm{N}$ protein ${ }^{6}$, but not in ST cells transfected with plasmids expressing $\mathrm{N}$ protein or infected with TGEV ${ }^{12}$. Overall, these data indicate that the presence of $\mathrm{N}$ protein in the nucleus of the infected cells might be of functional significance.

SARS-CoV ORF $3 \mathrm{~b}$ encodes a protein of 154 amino acids, lacking similarities to any known protein. Protein $3 \mathrm{~b}$ is predominantly localized in the nucleolus. A functional nuclear localization signal is located in amino acids 134 to 154 . Ectopic over-expression of protein $3 \mathrm{~b}$ in Vero, 293, and COS-7 cells induced cell cycle arrest at Go/G1 phase. ${ }^{13}$ EAV nsp1 also has been localized in the nucleus. ${ }^{10,14}$ Therefore, in total, at least three nidovirales proteins $(\mathrm{N}, 3 \mathrm{~b}$ and nsp 1 ) have been detected in the nucleus of infected cells, suggesting that nidovirales may modify cell behavior through the nucleus.

\subsection{CoV Genome Replication}

In CoV replication, recognition of RNA genome 5' and 3' ends by viral and cellular proteins is most likely essential. Furthermore, the interaction of these ends probably is a requirement for replication and transcription, as these are processes that must be initiated at the 3' end of the genome, and it has been shown that these processes are influenced by sequences mapping at the 5 ' end of the genome. ${ }^{15,16}$ There may be a direct interaction between the CoV 5' end 3' ends, as predicted for MHV and TGEV RNA genomes in the absence of protein using computer programs. ${ }^{17,18}$ Nevertheless, this direct interaction seems unlikely inside cells in the presence of cell and viral proteins. In fact, during genome synthesis, the first end synthesized (3') will be most likely immediately folded and nonspecifically covered by proteins, such as the $\mathrm{N}$ protein or nsp9, or by proteins binding specific RNA motifs with characteristic secondary structures. In fact, the 
postulated cross-talk between 5' and 3' ends and CoV replication has been shown in our laboratory. ${ }^{19}$ Precipitation of digoxigeninated 3'-ends by biotinylated 5'-ends using streptavidin sepharose beads and, the reverse (precipitation of digoxigeninated 5'-ends with biotinylated 3'-ends) have been shown. Cross-talk between the 5' and 3' ends of TGEV genome has been observed for $\mathrm{CoV}$ genome ends and only require the presence of cell proteins.

The $\mathrm{N}$ protein probably has a prominent role in $\mathrm{CoV}$ replication as it influences many viral and cellular processes. The role of the $\mathrm{N}$ protein is most likely constrained by its propensity to self-assemble to form the capsid and also by its phosphorylation state. $\mathrm{N}$ protein activity has to be a consequence of its interaction with other viral and cellular proteins and with virus and host cell nucleic acids. CoV N protein is associated with the replicase complex in double-membrane structures derived from the endoplasmic reticulum $^{20}$ and also binds to genome RNA forming the nucleocapsid. ${ }^{21-23}$ The nucleocapsid binds to the $\mathrm{M}$ protein carboxy-terminus in the endoplasmic reticulum (ER), Golgi and intermediate compartment (ERGIC) membranes. ${ }^{21,22,24,25}$ Particles bud as immature virions with annular large nucleocapsids. Immature virions are transported through the Golgi compartment, where a major rearrangement of the nucleocapsid takes place, giving rise to secretory vesicles containing mature virions with electrondense cores. $^{21,24,26}$

The $\mathrm{N}$ protein has a variable size in different CoVs (Fig. 1). Self-interactions of $\mathrm{N}$ proteins was first described in MHV. ${ }^{27}$ The $\mathrm{N}$ protein has conserved secondary structures, including highly conserved $\alpha$ helices, and a highly conserved serine-rich domain including the repetitive sequence SSDNSRSRSQSRSRSR ${ }^{12,28}$ (Fig. 1). Within this area, several active $\mathrm{N}$ protein domains have been mapped, such as the RNA binding domain of the IBV genome, ${ }^{29}$ the oligomerization binding domain (amino acids 184-196), ${ }^{30,31}$ and the $\mathrm{M}$ protein binding domain (amino acids 168-208), ${ }^{32}$ which is also part of the $\mathrm{N}$ protein oligomerization domain. These protein sequences may be crucial in maintaining the $\mathrm{N}$ protein in a correct conformation. In fact, deletion of the 168-208 aa region results in the complete loss of $\mathrm{N}$ protein dimerization.

Phosphorylation has been shown to cause conformational changes in MHV N protein structure. ${ }^{33}$ TGEV and IBV phosphoserine residues have been mapped within the CoV N protein primary and secondary structures. TGEV N protein serines 9, 156, 254, and 256 are phosphorylated in infected cells, ${ }^{12,28}$ while in IBV, N protein phosphorylation sites have been localized to serines $190,192,379$, and threonine $378 .^{29}$ CoVs N proteins present a conserved pattern of secondary structural elements, and a strong correlation has been observed between the MHV N protein three-domain organization and the predicted structure. N protein domains I and III are the most unstructured and divergent between $\mathrm{CoV}$, while domain II is more conserved. Interestingly, TGEV N serines 156, 254, and 256 were localized to domain II, adjacent to conserved secondary elements $\beta 3, \beta 6$, and $\alpha 7$, respectively. Therefore, phosphorylation in these serine residues could affect the structure of these secondary elements by the introduction of negative charges in a basic environment $^{34,35}$ and affect $\mathrm{N}$ protein RNA binding activity.

IBV $\mathrm{N}$ protein phosphorylation has been localized in sites distinct from those identified in TGEV, based on sequence comparisons. This apparent discrepancy could be explained by intrinsic differences between $\mathrm{CoV}$ species. The relevance of the identified TGEV N protein phosphoserines has been analyzed by site-directed mutagenesis using the TGEV infectious cDNA clone. ${ }^{36}$ Mutagenesis of all four TGEV phosphorylated 
serines to alanine did not prevent TGEV rescue from infectious cDNA nor lead to a significant TGEV titer reduction. This mutation may affect the binding of $\mathrm{CoV}$ N protein to RNA mediated by the amino terminus of this protein. ${ }^{29}$ Additional work is in progress to study the role of TGEV N protein phosphorylation.

The requirement of $\mathrm{N}$ protein for virus replication and transcription is debated. Certain observations suggest that $\mathrm{N}$ protein plays a role in replication, ${ }^{37-43}$ while others using either $\mathrm{CoV}^{44}$ or arterivirus systems ${ }^{45}$ claim that $\mathrm{N}$ protein is not essential. Using three TGEV derived replicons, two containing $\mathrm{N}$ gene in cis, and another one lacking this gene (Fig. 2), it has been clearly shown that TGEV replicon in the absence of $\mathrm{N}$ protein, provided either in cis or in trans, resulted in 50-fold greater levels of a reporter subgenomic RNA (gene 7) than background levels (Fig. 2). ${ }^{46}$ Interestingly, when N protein is provided in cis, replication-transcription increases 100 -fold over the levels in the absence of $\mathrm{N}$ protein. If $\mathrm{N}$ protein is exclusively provided in trans, replication-transcription levels increased 10-fold more (i.e., 1000-fold over levels in the absence of $\mathrm{N}$ protein). If $\mathrm{N}$ protein is in addition provided in cis, amplification levels do not increase over those reached when the $\mathrm{N}$ protein is only provided in trans. Two groups have shown that background levels of $\mathrm{CoV}$ transcription have been observed in the absence of $\mathrm{N}$ protein. ${ }^{43,46}$ Nevertheless, a substantial increase

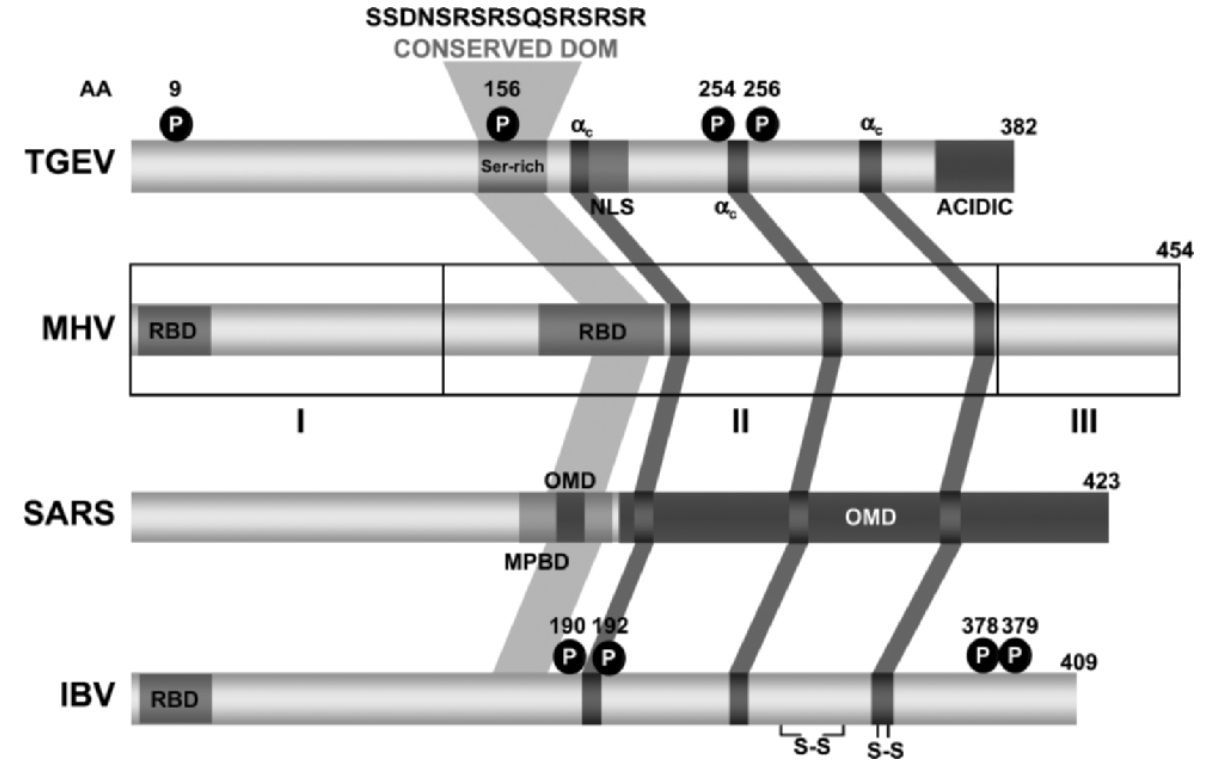

Figure 1. Scheme of $\mathrm{N}$ protein from different coronaviruses. The organization of $\mathrm{N}$ protein from four representative CoVs of genus $\alpha$ (TGEV), genus $\beta$ (MHV and SARS-CoV), and genus $\gamma$ (IBV) is indicated (not to scale). Conserved predicted structural elements are joined by gray shadowing zones. The three-domain organization proposed for MHV N protein by P. Master's group is indicated as open boxes over the MHV N protein (I, II, and III). P, phosphorylation sites. $\alpha \mathrm{c}$, protein domains with highly conserved alpha structure. AA, amino acid. NLS, nuclear localization signal. RBD, RNA binding domain. OMD, oligomerization domain. MPBD, M protein binding domain. S-S, disulfide bridge. 
in $\mathrm{CoV}$ transcription is observed by providing $\mathrm{N}$ protein either in cis or in trans. The increase in reporter gene expression could be due to an increase in the replication, in the transcription levels, or to both. There is a general agreement that the presence of $\mathrm{N}$ protein enhances the rescue of infectious virus from cDNA clones generated from different CoVs, such as IBV ${ }^{47}$ human coronavirus (HCoV)-229E, ${ }^{43,48}$ and TGEV using RNA in vitro transcripts ${ }^{49}$ or replicons. ${ }^{46}$

\section{CoV TRANSCRIPTION}

$\mathrm{CoV}$ transcription, and in general transcription in the Nidovirales order, is an RNAdependent RNA process which includes a discontinuous step during the production of subgenomic mRNAs. ${ }^{50,51}$ This transcription process ultimately generates a nested set of subgenomic mRNAs that are 5'- and 3'-coterminal with the virus genome. The common 5'-terminal leader sequence, 93 nucleotides (nt) in TGEV, is fused to the 5' end of the mRNA coding sequence (body) by a discontinuous transcription mechanism. Sequences preceding each gene represent signals for synthesis of subgenomic mRNAs (sgmRNAs).

\section{NO REP REP1 or REP2 REP 3}

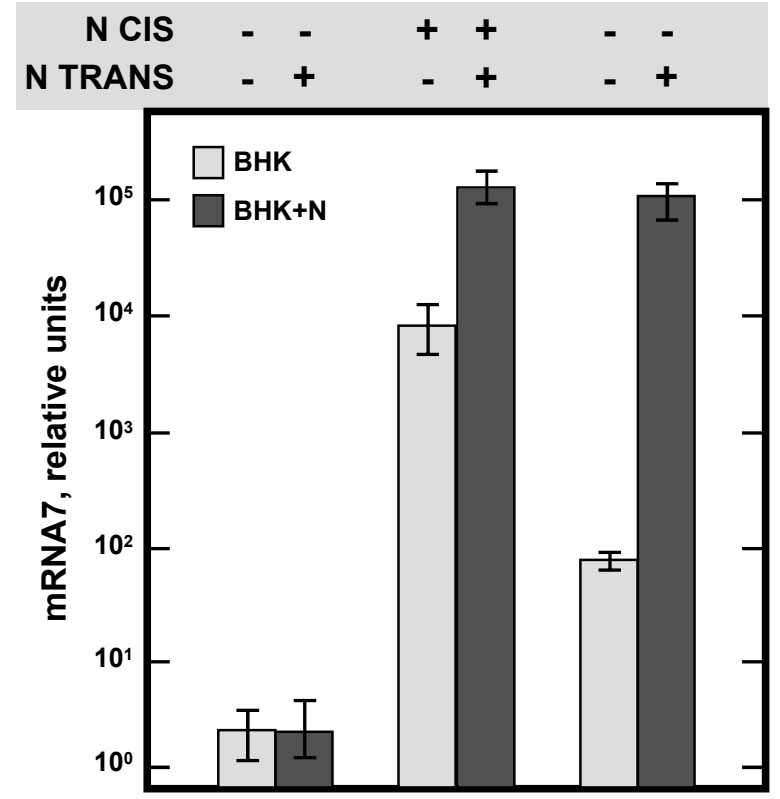

Figure 2. Expression of gene 7 in the presence of $\mathrm{N}$ protein. To study the effect of $\mathrm{N}$ protein in TGEV-derived replicon activity, the amount of mRNA7, expressed as relative units, was determined by real-time RT-PCR with specific oligonucleotides in RNA samples isolated from standard BHK-pAPN (BHK) or BHK-pAPN expressing $\mathrm{N}$ protein $(\mathrm{BHK}+\mathrm{N})$. Cells were transfected with either a non replicative cDNA clone (NO REP), two replicons that express the $\mathrm{N}$ protein (REP 1 and REP 2), or a replicon that does not encode protein N (REP 3). $\mathrm{N}$ protein was provided in cis by the replicons, or in trans using a Sinbis virus replicon when indicated (+). -, absence of $\mathrm{N}$ protein. The results indicate the mean values from three experiments, with standard deviations shown as error bars. 
These are the transcription-regulating sequences (TRSs) that include a conserved core sequence (CS; 5'-CUAAAC-3'), identical in all TGEV genes (CS-B), and the 5' and 3' flanking sequences ( 5 ' TRS and 3 ' TRS, respectively) that regulate transcription ${ }^{52}$ (Fig. 3 ). As this CS sequence is also found at the 3' end of the leader sequence (CS-L), it could base pair with the nascent minus strand complementary to each CS-B (cCS-B). In fact, the requirement for base pairing during transcription has been formally demonstrated in arteriviruses ${ }^{53,54}$ and CoVs by experiments in which base pairing between CS-L and the complement of CS-B was engineered in infectious genomic cDNAs. ${ }^{55}$ The canonical CS was nonessential for the generation of subgenomic mRNAs (sgmRNAs), but its presence led to transcription levels at least $10^{3}$-fold higher than its absence. The data obtained are compatible with a model of transcription that includes three steps (Fig. 3): (i) formation of 5'-3' complexes in the genomic RNA, (ii) scanning of base pairing of the nascent (-) RNA strand by the TRS-L, and (iii) template switch during synthesis of the negative strand to complete the (-) sgRNA. This template switch takes place after copying the CS sequence and was predicted in silico based on a high base pairing score between the nascent (-) RNA strand and the TRS-L.

The role in transcription of four nucleotides immediately flanking the CS both at the 5 ' and 3 ' ends has been studied using a transcriptionally inactive canonical CS (CS-S2) internal to the $\mathrm{S}$ gene. ${ }^{56}$ The rationale for selecting 5' and 3' TRS flanking sequences consisting of four nucleotides comes from the results of an in silico analysis showing that to predict both viral mRNAs and alternative mRNAs at noncanonical junction sites, an optimal TRS-L should include the CS plus four nucleotides flanking the CS at both ends. These predictions have been supported by experimental data performed by reverse genetic analysis of the sequences immediately flanking CS-S2. A good correlation was observed between the free energy of the TRS-L and cTRS-B duplex formation and the levels of subgenomic mRNA-S2, demonstrating that base pairing between leader and body beyond the CS was a determinant in the regulation of CoV transcription. In TRS mutants with increasing complementarity between TRS-L and cTRS-B, a tendency to reach a plateau in $\Delta \mathrm{G}$ values was observed, suggesting that a more precise definition of the TRS limits might be proposed, consisting of the central CS and approximately four nucleotides flanking 5' and 3' the CS. Sequences downstream of the CS exert a stronger influence on the template-switching decision in accordance with a model of polymerase strand-transfer and template-switching during minus strand synthesis.

According to the working model of transcription proposed by our laboratory (Fig. 3), the first step is the interaction of the leader TRS with a complex presumably formed by the replicase, the helicase, the nascent RNA of negative polarity, and other viral and cellular proteins involved in transcription. Candidate proteins have been reported by several laboratories. On the viral side, essential proteins in transcription are the RNAdependent RNA-polymerse (RdRp), and the helicase (Hel). In addition, N protein probably increases basal transcription (see above) and nspl has clearly been involved in arterivirus transcription. ${ }^{57,58}$ It has also been suggested that NendoU may play a role by specifically cutting double stranded RNA generated (transcriptive intermediates) during the synthesis of the nascent RNA of negative polarity. NendoU nuclease has a strong preference for cleavage at $\mathrm{GU}(\mathrm{U})$ sequences in double-stranded RNA substrates. ${ }^{59,60}$ It has been suggested that the GU(U) sequence at the 3' terminus of nascent minus-strand RNAs, which corresponds to conserved AAC nucleotides in the core of the CoV gene TRSs elements, might be substrate of this activity; therefore, NendoU activity might be 
involved in the transcription of subgenomic mRNAs. Data from our laboratory in which we analyzed around 90 different sgRNAs generated during the mutagenesis of a TGEV $\mathrm{CS},{ }^{55}$ and also from other laboratories, ${ }^{43}$ support the functional relevance of the AAC sequence in transcription, but further studies are required to provide a direct link to the activities of enzymes such as the uridylate-specific endoribonuclease.

In addition, cell proteins most likely play a role in $\mathrm{CoV}$ transcription regulation. In fact, there are data directly involving heterogeneous nuclear ribonucleoprotein (hnRNP) $\mathrm{A} 1,{ }^{61,62}$ hnRNP I (PTB), ${ }^{63}$ and the elongation factor eEF-1 in CoV transcription. ${ }^{64,65}$ Furthermore, proteins such as p100 kDa coactivator and annexin A2 may be involved in $\mathrm{CoV}$ transcription as we have shown that these proteins bind to TRS sequences. ${ }^{56}$ In the arteriviruses, the p100 kDa coactivator interacts with nsp 1 involved in transcription and may regulate this activity. ${ }^{58}$
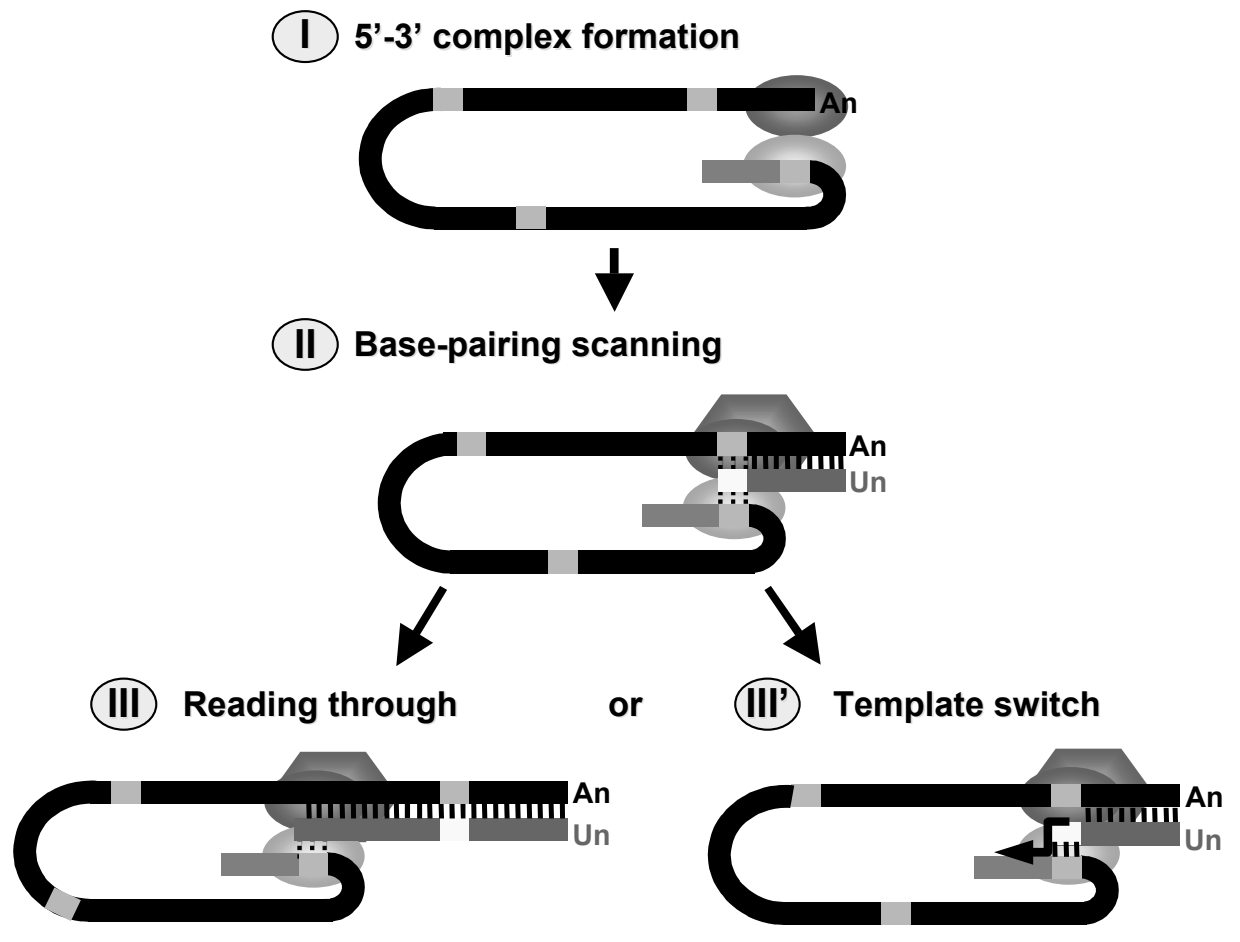

Figure 3. Three-step working model of coronavirus transcription. (I) 5'-3' complex formation step. Proteins binding the 5' and 3' end TGEV sequences are represented by ellipsoids. Leader sequence is indicated with dark gray bars, CS sequences are indicated with a clear bar. An, poly A tail. (II) Base pairing scanning step. Minus strand RNA is in a lighter color compared with positive-strand RNA. The transcription complex is represented by the hexagon. Vertical dotted bars represent scanning of base pairing by the TRS-L sequence in the transcription process. Vertical solid bars indicate complementarity between the genomic (gRNA) and the nascent minus strand. Un, poly U tail. (III) The synthesis of the negative strand can continue to make a longer sgRNA (III left), or a template switch step can take place (III right) as indicated in the text. The thick arrow indicates the switch in the template made by the transcription complex to complete the synthesis of (-) sgRNA. 
$\mathrm{CoV}$ genomic RNA interacts with at least three proteins: hnRNP A1, ${ }^{61,62,66,67}$ PTB, ${ }^{68,69}$ and $\mathrm{N}^{37,42,70}$ and may mediate the formation of complexes between the leader TRS and the transcription complex at the body TRS. Formation of cyclic complexes could in principle be mediated by the interaction among these proteins. In fact, binding between hnRNPA1 and PTB, ${ }^{71,72}$ hnRNP A1 and N protein, ${ }^{73,74}$ and PTB and N protein ${ }^{63}$ have been documented. Many of these biochemical interactions have been reported in the past eight years and their role can now be reinterpreted in the context of the transcription model implying discontinuous RNA synthesis during the production of the negative strand. A role in transcription has been assigned to different proteins:

(i) hnRNPA1 protein binds to the complementary strand (negative-polarity) of the MHV leader (cL) and to TRS sequences, particularly the consensus (3'-AGAUUUG-5') sequence of MHV RNA located at the 3' end of the genome. ${ }^{62}$ Site-specific mutations of TRSs inhibited the mRNA transcription from MHV DI RNA, in direct proportion to the extent of reduction of hnRNPA1 binding to $\mathrm{cL} .{ }^{62,75}$ The effect of hnRNP A1 on MHV RNA transcription was further confirmed in cell lines. ${ }^{67}$ Direct evidence for a functional role for hnRNP A1 in MHV synthesis has been demonstrated in MHV-infected cells. ${ }^{76}$ Binding of hnRNP A1 to a TRS also correlates with the efficiency of transcription from that TRS. ${ }^{62,75}$ In addition, a C-terminus-truncated hnRNPA1 mutant exhibited dominantnegative effects on viral genomic RNA replication and subgenomic transcription. Therefore, hnRNP A1 may regulate CoV RNA-dependent transcription.

(ii) The N protein of MHV binds the UCUAAAC sequence of the leader RNA, and it has been suggested that $\mathrm{N}$ protein is involved in MHV RNA transcription. ${ }^{37,42}$ The role of $\mathrm{N}$ protein in MHV RNA replication has also been shown in an in vivo replication system. ${ }^{38}$ These findings suggest that both cellular hnRNPA1 and viral $\mathrm{N}$ protein are components of the MHV replication and transcription complex. As hnRNPA1 interacts with some serine-arginine (SR)-rich proteins, ${ }^{73}$ and because $\mathrm{N}$ protein also contains an SR motif, ${ }^{77,78}$ it has been proposed that hnRNPA1 interacts directly with $\mathrm{N}$ protein to bring the leader RNA to the CS sequence of the template RNA for initiation of subgenomic mRNA transcription. In fact, it has been shown ${ }^{74}$ that $\mathrm{N}$ protein interacts with hnRNPA1 both in vivo and in vitro. The data was confirmed using the two-hybrid system. In agreement with these results, we have shown by Far-Western blotting that TGEV N protein binds PTB. ${ }^{79}$

Template switch during transcription may be aided by the chaperone activity of $\mathrm{CoV}$ $\mathrm{N}$ protein. During negative-strand RNA synthesis, a template switch is required to add a negative copy of the leader to the negative strand. This process represents a displacement of the former template RNA by another one, the leader sequence. These types of processes need to overcome an energy barrier threshold. RNA chaperones are RNA binding proteins that may help to overcome this threshold. We have shown that TGEV N protein is an RNA chaperone that is also active in viral RNA annealing (Zuñiga et al. in this book $^{79}$ ).

(iii) PTB binds to the c3'-untranslated region (UTR) of MHV inducing a conformational change in RNA structure. ${ }^{68}$ Mutations of the PTB-binding site in either 5'-leader or the sequences complementary to 3'-UTR inhibited replication and transcription of MHV genomic and defective-interfering (DI) RNA, in direct proportion to the extent of reduction of PTB binding, suggesting that PTB plays a role in regulating viral RNA synthesis. Thus, the interaction of $\mathrm{N}$ protein with PTB may modulate transcription. ${ }^{63}$ 
(iv) SYNCRIPT (p70) is a member of the hnRNP family and localizes largely in the cytoplasm. The p70 cross-linked to MHV positive- or negative-strand RNA. The p70binding site was mapped to the leader sequence of the 5'-UTR, requiring the UCUAA repeat sequence. Overexpression of p70 inhibited syncytium formation induced by MHV. Furthermore, downregulation of the endogenous $\mathrm{p} 70$ with a specific short iRNA delayed MHV RNA synthesis. These results suggest that p70 may be directly involved in MHV RNA replication as a positive regulator. ${ }^{8}$

(v) EAV nspl has been proposed to couple genome replication and transcription. ${ }^{81}$ Nsp-1 has been shown to interact with p100, ${ }^{58}$ and nsp1-p100 interactions have been speculated to be important for viral sgRNA synthesis, either directly or by recruiting a p100 binding protein to the viral RdRp complex. Alternatively, nsp1 might modulate transcription in the infected cell, explaining why the protein is targeted to the nucleus. ${ }^{10}$

\section{CONCLUSIONS}

The precise role of the described protein and other viral and cellular proteins needs to be confirmed in the context of discontinuous transcription during the synthesis of the negative strand, giving special attention to intermediates of the replicase processing and to proteins associated with membrane structures located in the cytoplasm. Functional proteomics could be of great help in this complicated task. In addition, the establishment of in vitro replication and transcription systems will help to clarify the mechanisms involved in $\mathrm{CoV}$ replication.

We thank D. Dorado and M. González for technical assistance. This work was supported by grants from the Comisión Interministerial de Ciencia y Tecnología (CICYT) of the Department of Education and Science (Spain), Fort Dodge Veterinaria and the European Communities (Frame VI Life Sciences, projects DISSECT ref. SP22CT-2004-511060, and TIPP ref. QLK2-CT-2002-01050). CC has received a fellowship from the Education Department of the Community of Madrid.

\section{REFERENCES}

1. M. Carmo-Fonseca, L. Mendes-Soares, and I. Campos, To be or not to be in the nucleolus, Nat. Cell Biol. 2 , E107-E112 (2002)

2. J. A. Hiscox, The nucleolus - a gateway to viral infection? Arch. Virol. 147, 1077-1089 (2002).

3. M. O. Olson, M. Dundr, and A. Szebeni, The nucleolus: an old factory with unexpected capabilities, Trends Cell Biol. 10, 189-196 (2000).

4. T. Pederson, The plurifunctional nucleolus, Nucleic Acids Res. 26, 3871-3876 (1998).

5. T. Pederson and J. C. Politz, The nucleolus and the four ribonucleoproteins of translation, J. Cell Biol. 148, 1091-1095 (2000).

6. T. Wurm, H. Chen, T. Hodgson, P. Britton, G. Brooks, and J. A. Hiscox, Localization to the nucleolus is a common feature of coronavirus nucleoproteins, and the protein may disrupt host cell division, J. Virol. 75, 9345-9356 (2001).

7. J. H. You, B. K. Dove, G. Howell, P. Heinen, M. Zambon, and J. A. Hiscox, Sub-cellular localisation of the severe acute respiratory syndrome coronavirus viral RNA binding protein, nucleoprotein, J. Gen. Virol. in press (2005)

8. K. A. Timani, L. Ye, Y. Zhu, Z. Wu, and Z. Gong, Cloning, sequencing, expression, and purification of SARS-associated coronavirus nucleocapsid protein for serodiagnosis of SARS, J. Clin. Virol. 30, 309-312 (2004). 
9. H. Chen, B. Coote, S. Attree, and J. A. Hiscox, Evaluation of a nucleoprotein-based enzyme-linked immunosorbent assay for the detection of antibodies against infectious bronchitis virus, Avian Pathol. 32 , 519-526 (2003).

10. M. A. Tijms, Y. van der Meer, and E. J. Snijder, Nuclear localization of non-structural protein 1 and nucleocapsid protein of equine arteritis virus, J. Gen. Virol. 83, 795-800 (2002).

11. R. R. Rowland, R. Kervin, C. Kuckleburg, A. Sperlich, and D. A. Benfield, The localization of porcine reproductive and respiratory syndrome virus nucleocapsid protein to the nucleolus of infected cells and identification of a potential nucleolar localization signal sequence, Virus Res. 64, 1-12 (1999).

12. E. Calvo, D. Escors, J. A. Lopez, et al., Phosphorylation and subcellular localization of transmissible gastroenteritis virus nucleocapsid protein in infected cells, J. Gen. Virol. 86, 2255-2267 (2005)

13. X. Yuan, Z. Yao, Y. Shan, et al., Nucleolar localization of non-structural protein $3 b$, a protein specifically encoded by the severe acute respiratory syndrome coronavirus, Virus Res. in press (2005).

14. Y. van der Meer, E. J. Snijder, J. C. Dobbe, et al., Localization of mouse hepatitis virus nonstructura proteins and RNA synthesis indicates a role for late endosomes in viral replication, J. Virol. 73, 7641-7657 (1999).

15. R. van der Most, W. Luytjes, S. Rutjes, and S. J. M. Spaan, Translation but not the encoded sequence is essential for the efficient propagation of the defective interfering RNAs of the coronavirus mouse hepatitis virus, J. Virol. 69, 3744-3751 (1995).

16. Y.-J. Lin, C. L. Liao, and M. M. C. Lai, Identification of the cis-acting signal for minus-strand RNA synthesis of a murine coronavirus: implications for the role of minus-strand RNA in RNA replication and transcription, J. Virol. 68, 8131-8140 (1994).

17. J. Holt, J. Y. Sgro, M. Zuker, and A. Palmenberg, Computer folding of full-length viral genomes: a new toolkit for automated analysis of RNAs longer than 10,000 bases, Seventh International Symposium on positive strand RNA viruses, San Francisco, California, USA (2004).

18. J. Y. Sgro, J. Holt, M. Zuker, and A. Palmenberg, RNA folding of the complete SARS and MHV coronavirus genomes, Seventh International Symposium on positive strand RNA viruses, San Francisco, California, USA (2004).

19. C. Galan, F. Almazan, and L. Enjuanes, Cross-talk between the 5'- and 3'-ends of coronavirus genome, submitted (2005).

20. R. Gosert, A. Kanjanahaluethai, D. Egger, K. Bienz, and S .C. Baker, RNA replication of mouse hepatitis virus takes place at double-membrane vesicles, J. Virol. 76, 3697-3708 (2002).

21. D. Escors, J. Ortego, H. Laude, and L. Enjuanes, The membrane M protein carboxy terminus binds to transmissible gastroenteritis coronavirus core and contributes to core stability, J. Virol. 75, 1312-1324 (2001).

22. K. Narayanan, A. Maeda, J. Maeda, and S. Makino, Characterization of the coronavirus M protein and nucleocapsid interaction in infected cells, J. Virol. 74, 8127-8134 (2000).

23. L. S. Sturman, K. V. Holmes, and J. Behnke, Isolation of coronavirus envelope glycoproteins and interaction with the viral nucleocapsid, J. Virol. 33, 449-462 (1980).

24. I. J. Salanueva, J. L. Carrascosa, and C. Risco, Structural maturation of the transmissible gastroenteritis coronavirus, J. Virol. 73, 7952-7964 (1999).

25. C. Risco, M. Muntión, L. Enjuanes, and J. L. Carrascosa, Two types of virus-related particles are found during transmissible gastroenteritis virus morphogenesis, J. Virol. 72, 4022-4031 (1998).

26. J. Ortego, D. Escors, H. Laude, and L. Enjuanes, Generation of a replication-competent, propagationdeficient virus vector based on the transmissible gastroenteritis coronavirus genome, J. Virol. 76, 1151811529 (2002).

27. S. G. Robbins, M. F. Frana, J. J. McGowan, J. F. Boyle, and K. V. Holmes, RNA-binding proteins of coronavirus MHV: detection of monomeric and multimeric N protein with an RNA overlay-protein blot assay, Virology 150, 402-410 (1986).

28. P. Britton, Coronavirus motif, Nature 353, 394 (1991).

29. H. Chen, A. Gill, B. K. Dove, et al., Mass spectroscopic characterization of the coronavirus infectious bronchitis virus nucleoprotein and elucidation of the role of phosphorylation in RNA binding by using surface plasmon resonance, J. Virol. 79, 1164-1179 (2005).

30. R. He, F. Dobie, M. Ballantine, et al., Analysis of multimerization of the SARS coronavirus nucleocapsid protein, Biochem. Biophys. Res. Commun. 316, 476-483 (2004).

31. M. Surjit, B. Liu, S. Jameel, V. T. Chow, and S. K. Lal, The SARS coronavirus nucleocapsid protein induces actin reorganization and apoptosis in COS-1 cells in the absence of growth factors, Biochem. J. 383, 13-18 (2004).

32. R. He, A. Leeson, M. Ballantine, et al., Characterization of protein-protein interactions between the nucleocapsid protein and membrane protein of the SARS coronavirus, Virus Res. 105, 121-125 (2004). 
33. S. A. Stohlman, J. O. Fleming, C. D. Patton, and M. M. C. Lai, Synthesis and subcellular localization of the murine coronavirus nucleocapsid protein, Virology 130, 527-532 (1983).

34. M. M. Parker and P. S. Masters, Sequence comparison of the N genes of five strains of the coronavirus mouse hepatitis virus suggests a three domain structure for the nucleocapsid protein, Virology 179, 463-468 (1990).

35. P. S. Masters, Localization of an RNA-binding domain in the nucleocapsid protein of the coronavirus mouse hepatitis virus, Arch. Virol. 125, 141-160 (1992).

36. E. Alvarez, M. L. DeDiego, D. Escors, and L. Enjuanes, Role of transmissible gastroenteritis Coronavirus N protein phosphorylation in virus replication, submitted (2005).

37. R. S. Baric, G. W. Nelson, J. O. Fleming, et al., Interactions between coronavirus nucleocapsid protein and viral RNAs: implications for viral transcription, J. Virol. 62, 4280-4287 (1988).

38. S. R. Compton, D. B. Rogers, K. V. Holmes, D. Fertsch, J. Remenick, and J. J. McGowan, In vitro replication of mouse hepatitis virus strain A59, J. Virol. 69, 2313-2321 (1987)

39. Y.-N. Kim and S. Makino, Characterization of a murine coronavirus defective interfering RNA internal cisacting replication signal, J. Virol. 69, 4963-4971 (1995).

40. H. Laude and P. S. Masters, in: The Coronaviridae, edited by S. G. Siddell (Plenum Press, New York, 1995) pp. 141-158.

41. G. W. Nelson, S. A. Stohlman, and S. M. Tahara, High affinity interaction between nucleocapsid protein and leader/intergenic sequence of mouse hepatitis virus RNA, J. Gen. Virol. 81, 181-188 (2000).

42. S. A. Stohlman, R. S. Baric, G. N. Nelson, L. H. Soe, L. M. Welter, and R. J. Deans, Specific interaction between coronavirus leader RNA and nucleocapsid protein, J. Virol. 62, 4288-4295 (1988).

43. B. Schelle, N. Karl, B. Ludewig, S. G. Siddell, and V. Thiel, Selective replication of coronavirus genomes that express nucleocapsid protein, J. Virol. 79, 6620-6630 (2005).

44. V. Thiel, J. Herold, B. Schelle, and S. G. Siddell, Viral replicase gene products suffice for coronavirus discontinuous transcription, J. Virol. 75, 6676-6681 (2001).

45. R. Molenkamp, H. van Tol, B. C. Rozier, Y. van der Meer, W. J. Spaan, and E. J. Snijder, The arterivirus replicase is the only viral protein required for genome replication and subgenomic mRNA transcription, J. Gen. Virol. 81, 2491-2496 (2000).

46. F. Almazan, C. Galan, and L. Enjuanes, The nucleoprotein is required for efficient coronavirus genome replication, J. Virol. 78, 12683-12688 (2004).

47. R. Casais, V. Thiel, S. G. Siddell, D. Cavanagh, and P. Britton, Reverse genetics system for the avian coronavirus infectious bronchitis virus, J. Virol. 75, 12359-12369 (2001).

48. V. Thiel, N. Karl, B. Schelle, P. Disterer, I. Klagge, and S. G. Siddell, Multigene RNA vector based on coronavirus transcription, J. Virol. 77, 9790-9798 (2003).

49. K. M. Curtis, B. Yount, and R. S. Baric, Heterologous gene expression from transmissible gastroenteritis virus replicon particles, J. Virol. 76, 1422-1434 (2002).

50. S. G. Sawicki and D. L. Sawicki, A new model for coronavirus transcription, Adv. Exp. Med. Biol. 440, $215-$ 220 (1998).

51. M. M. C. Lai and D. Cavanagh, The molecular biology of coronaviruses, Adv. Virus Res. 48, 1-100 (1997).

52. S. Alonso, A. Izeta, I. Sola, and L. Enjuanes, Transcription regulatory sequences and mRNA expression levels in the coronavirus transmissible gastroenteritis virus, J. Virol. 76, 1293-1308 (2002).

53. A. O. Pasternak, E. van den Born, W. J. M. Spaan, and E. J. Snijder, Sequence requirements for RNA strand transfer during nidovirus discontinuous subgenomic RNA synthesis, EMBO J. 20, 7220-7228 (2001).

54. G. van Marle, J. C. Dobbe, A. P. Gultyaev, W. Luytjes, W. J. M. Spaan, and E. J. Snijder, Arterivirus discontinuous mRNA transcription is guided by base pairing between sense and antisense transcriptionregulating sequences, Proc. Natl. Acad. Sci. USA 96, 12056-12061 (1999).

55. S. Zúñiga, I. Sola, S. Alonso, and L. Enjuanes, Sequence motifs involved in the regulation of discontinuous coronavirus subgenomic RNA synthesis, J. Virol. 78, 980-994 (2004).

56. I. Sola, J. L. Moreno, S. Zúñiga, S. Alonso, and L. Enjuanes, Role of nucleotides immediately flanking the transcription-regulating sequence core in coronavirus subgenomic mRNA synthesis, J. Virol. 79, 25062516 (2005).

57. L. C. van Dinten, J. A. den Boon, A. L. M. Wassenaar, W. J. M. Spaan, and E. J. Snijder, An infectious arterivirus cDNA clone: identification of a replicase point mutation that abolishes discontinuous mRNA transcription, Proc. Natl. Acad. Sci. USA 94, 991-996 (1997).

58. M. A. Tijms and E. J. Snijder, Equine arteritis virus non-structural protein 1, an essential factor for viral subgenomic mRNA synthesis, interacts with the cellular transcription co-factor p100, J. Gen. Virol. 84, 2317-2322 (2003).

59. K. A. Ivanov, T. Hertzig, M. Rozanov, et al., Major genetic marker of nidoviruses encodes a replicative endoribonuclease, Proc. Natl. Acad. Sci. USA 101, 12694-12699 (2004) 
60. E. J. Snijder, P. J. Bredenbeek, J. C. Dobbe, et al., Unique and conserved features of genome and proteome of SARS-coronavirus, and early split-off from the coronavirus group 2 lineage, J. Mol. Biol. 331, 991-1004 (2003).

61. G. Zhang, V. Slowinski, and K. A. White, Subgenomic mRNA regulation by a distal RNA element in a $(+)$ strand RNA virus, RNA 5, 550-561 (1999).

62. H. P. Li, X. Zhang, R. Duncan, L. Comai, and M. M. C. Lai, Heterogeneous nuclear ribonucleoprotein A1 binds to the transcription-regulatory region of mouse hepatitis virus RNA, Proc. Natl. Acad. Sci. USA 94, 9544-9549 (1997).

63. K. S. Choi, P. Huang, and M. M. Lai, Polypyrimidine-tract-binding protein affects transcription but not translation of mouse hepatitis virus RNA, Virology 303, 58-68 (2002).

64. S. Banerjee, K. Narayanan, T. Mizutani, and S. Makino, Murine coronavirus replication-induced p38 mitogen-activated protein kinase activation promotes interleukin-6 production and virus replication in cultured cells, J. Virol. 76, 5937-5948 (2002).

65. T. Mizutani, S. Fukushi, M. Saijo, I. Kurane, and S. Morikawa, Phosphorylation of p38 MAPK and its downstream targets in SARS coronavirus-infected cells, Biochem. Biophys. Res. Commun. 319, 1228-1234 (2004).

66. T. Furuya and M. M. C. Lai, Three different cellular proteins bind to complementary sites on the 5'-endpositive and 3'-end negative strands of mouse hepatitis virus RNA, J. Virol. 67, 7215-7222 (1993).

67. S. T. Shi, P. Huang, H. P. Li, and M. M. C. Lai, Heterogeneous nuclear ribonucleoprotein A1 regulates RNA synthesis of a cytoplasmic virus, EMBO J. 19, 4701-4711 (2000).

68. P. Huang and M. M. C. Lai, Polypyrimidine tract-binding protein binds to the complementary strand of the mouse hepatitis virus 3' untranslated region, thereby altering RNA conformation, J. Virol. 73, 9110-9116 (1999).

69. H.-P. Li, P. Huang, S. Park, and M. M. C. Lai, Polypyrimidine tract-binding protein binds to the leader RNA of mouse hepatitits virus and serves as a regulator of viral transcription, J. Virol. 73, 772-777 (1999).

70. C. Galan, L. Enjuanes, and F. Almazan, A point mutation within the replicase gene differentially affects coronavirus genome versus minigenome replication, submitted (2005).

71. A. L. Bothwell, D. W. Ballard, W. M. Philbrick, et al., Murine polypyrimidine tract binding protein. Purification, cloning, and mapping of the RNA binding domain, J. Biol. Chem. 266, 24657-24663 (1991).

72. M. M. C. Lai, Cellular factors in the transcription and replication of viral RNA genomes: a parallel to DNAdependent RNA transcription, Virology 244, 1-12 (1998).

73. L. Cartegni, M. Maconi, E. Morandi, F. Cobianchi, S. Riva, and G. Biamonti, hnRNP A1 selectively interacts through its Gly-rich domain with different RNA-binding proteins, J. Mol. Biol. 59, 337-348 (1996).

74. Y. Wang and X. Zhang, The nucleocapsid protein of coronavirus mouse hepatitis virus interacts with the cellular heterogeneous nuclear ribonucleoprotein A1 in vitro and in vivo, Virology 265, 96-109 (1999).

75. X. Zhang and M. M. C. Lai, Interactions between the cytoplasmic proteins and the intergenic (promoter) sequence of mouse hepatitis virus RNA: correlation with the amounts of subgenomic mRNA transcribed, $J$. Virol. 69, 1637-1644 (1995).

76. X. Zhang, H. P. Li, W. Xue, and M. M. C. Lai, Formation of a ribonucleoprotein complex of mouse hepatitis virus involving heterogeneous nuclear ribonucleoprotein A1 and transcription-regulatory elements of viral RNA, Virology 264, 115-124 (1999).

77. D. Peng, C. A. Koetzner, and P. S. Masters, Analysis of second-site revertants of a murine coronavirus nucleocapsid protein deletion mutant and construction of nucleocapsid protein mutants by targeted RNA recombination, J. Virol. 69, 3449-3457 (1995).

78. D. Peng, C. A. Koetzner, T. McMahon, Y. Zhu, and P. Masters, Construction of murine coronavirus mutants containing interspecies chimeric nucleocapsid proteins, J. Virol. 69, 5475-5484 (1995).

79. S. Zúñiga, I. Sola, J. L. Moreno, and L. Enjuanes, Coronavirus nucleocapsid protein is an RNA chaperone. Implications for transcription regulation, submitted (2005).

80. K. S. Choi, A. Mizutani, and M. M. C. Lai, SYNCRIP, a member of the heterogeneous nuclear ribonucleoprotein family, is involved in mouse hepatitis virus RNA synthesis, J. Virol. 78, 13153-13162 (2004).

81. M. A. Tijms, L. C. van Dinten, A. E. Gorbalenya, and E. J. Snijder, A zinc finger-containing papain-like protease couples subgenomic mRNA synthesis to genome translation in a positive-stranded RNA virus, Proc. Natl. Acad. Sci. USA 98, 1889-1894 (2001). 